\title{
Relaxed computation of non-bifurcation progressive disaster recovery problem
}

\author{
Shohei Kamamura ${ }^{\text {a) }}$, Yoshihiko Uematsu, and Kouichi Genda \\ NTT Network Service Systems Laboratories, NTT Corporation, \\ 3-9-11 Midori-cho, Musashino-shi, Tokyo 466-8555, Japan \\ a)kamamura.shohei@lab.ntt.co.jp
}

\begin{abstract}
We propose relaxed computation for the non-bifurcation progressive disaster recovery problem. When massive failure occurs, failed components are gradually repaired since repair resources are limited. Though there are studies on disaster recovery problem to maximize the amount of recovered traffic considering this assumption, they are based on the maximum flow approach, where traffic bifurcation on an arbitrary node is allowed. This condition is not practical in an actual environment. We first formulate non-bifurcation progressive disaster recovery problem as $0-1$ integer linear programming. Because the problem is NP-hard, we present a problem-decomposition method and obtain an improvement of $13 \%$ over the benchmark method.
\end{abstract}

Keywords: disaster recovery, integer linear programming, multi-commodity flow, non-bifurcation

Classification: Network System

\section{References}

[1] J. Wang, C. Qiao, and H. Yu, "On progressive network recovery after a major disruption," Proc. IEEE INFOCOM, Shanghai, pp. 1925-1933, April 2011. DOI:10.1109/INFCOM.2011.5934996

[2] K. Genda, H. Yamamoto, and S. Kamamura, "Fast and traffic-balanced network recovery from massive failures," IEICE Commun. Express, vol. 4, no. 8, pp. 264-269, 2015. DOI:10.1587/comex.4.264

[3] S. Kamamura, D. Shimazaki, Y. Uematsu, K. Genda, and K. Sasayama, "Multistaged network restoration from massive failures considering transition risks," Proc. of IEEE ICC 2014, pp. 1308-1313, 2014. DOI:10.1109/ICC.2014. 6883502

[4] Y. Wang and Z. Wang, "Explicit routing algorithms for internet traffic engineering," Eight International Conference on Computer Communications and Networks, 1999. Proc., pp. 582-588, Oct. 1999. DOI:10.1109/ICCCN. 1999.805577

[5] R. M. Karp, "Reducibility among combinatorial problems," in Complexity of Computer Computations, pp. 85-103, Plenum, New York, 1972. DOI:10.1007/ 978-1-4684-2001-2_9

[6] H. Matsuura, "Multipath creation algorithm optimizing traffic dispersion on networks," Proc. of APNOMS 2011, pp. 1-8, Sept. 2011. DOI:10.1109/ APNOMS.2011.6077016 


\section{Introduction}

Because disaster disrupts many network resources (links and nodes), conventional recovery mechanisms such as transport protection or upper-layer restoration would not work well with insufficient physical resources. Therefore, rapid repairing of not only communication paths but also failed resources is required. Wang et al. proposed the progressive disaster recovery concept [1] for massive failure recovery, where failed resources can be gradually repaired since the availability of repair resources, e.g., physical and human, is limited. Then, they formulated the progressive disaster recovery problem as mixed integer linear programming (MILP) for maximizing recovered traffic involving transient repairing stages. As related works, we proposed a method for improving the above method. Ref. [2] considered fairness between flows, and Ref. [3] considered transition risks caused by the path switching operation.

The existing methods $[1,2,3]$, which use a mathematical programming approach, are based on the maximum flow approach, where traffic bifurcation on an arbitrary node is allowed. For an actual transport network environment, however, traffic bifurcation is only allowed on the source node for the purpose of protection; traffic bifurcation for load balancing is not practical. By formulating the progressive disaster recovery problem as $0-1$ integer linear programming (ILP) based on the multi-commodity flow problem [4], we can define the non-bifurcation problem. However, 0-1 ILP is classified as class NP-hard, and it cannot yield solutions within a practical amount of time. Therefore, our goal is to solve the non-bifurcation progressive disaster recovery problem within a practical amount of time.

We formulate the non-bifurcation progressive disaster recovery problem as $0-1$ ILP based on the multi-commodity flow problem, and we propose the problemdecomposition method for simply solving the problem. The method first divides the problem into two problems: the physical resource repairing order decision problem and failed path computation problem. This division is based on our hypothesis: if each divided problem has the same objective function, the performance degradation caused by the problem decomposition is small. Next, the failed path computation problem is divided into the path configuration order decision problem and single path route computation problem. This is based on the fact that the path configuration order for recovery often accords with the network operation policy, e.g., quality of services and/or service level agreement. Every decomposed problem can be solved by simple heuristic algorithms within polynomial time. As a result, our method achieves an improvement of $13 \%$ over the benchmark method.

There are two contributions in this paper. To the best of our knowledge, we are the first to provide the progressive disaster recovery problem as based on the multicommodity flow problem, which does not allow traffic bifurcation. The second is a simple and fast computation method by problem decomposition. In section 2, we present the formulation of the problem, and we report our problem decomposition method and numerical examples in section 3. 


\section{Problem formulation}

In this section, we provide the formulation of the progressive disaster recovery problem as 0-1 ILP based on the multi-commodity flow problem. The network is given as a graph $G=(V, E)$, where $V$ is a set of physical nodes (buildings), and $E$ is a set of physical links. Capacity $C_{i j}$ is given for each link $(i, j) \in E$.

Our important assumption is that failed resources can be gradually repaired since the availability of repair resources is limited. Then, repairing process is staged: a certain amount of resources is repaired in one stage. We assumed that failed resources are gradually repaired through $K$ times repairing stages. The value $y_{i j}^{i n i t}$, the binary value, indicates the condition of a physical link $(i, j)$ at the first state. If link $(i, j)$ is disrupted at the first state, $y_{i j}^{i n i t}$ becomes 0 . Otherwise, $y_{i j}^{i n i t}$ becomes 1 . Node failure is represented by multiple link failure, which connects the node. Traffic demand between the node pair $p \in P$ is given as $d^{p}$. The variables are $x_{i j k}^{p}, y_{i j k}$, and $\alpha_{k}$. If the traffic between $p$ uses link $(i, j)$ at stage $k, x_{i j k}^{p}$ becomes 1 . Otherwise, $x_{i j k}^{p}$ becomes 0 . If link $(i, j)$ is repaired at stage $k, y_{i j k}$ becomes 1 . Otherwise, $y_{i j k}$ becomes $0 . \alpha_{k}$ represents the maximum link utilization at stage $k$. By using the above notations, our problem is formulated as follows.

$$
\min \sum_{k=0}^{K} \alpha_{k}
$$

s. t.

$$
\begin{gathered}
\sum_{j:(i, j) \in E} x_{i j k}^{p}-\sum_{j:(j, i) \in E} x_{i j k}^{p}= \begin{cases}1 & \text { if } \mathrm{i}=\text { source } \\
-1 & \text { if } \mathrm{i}=\text { destination } \\
0 \quad \text { otherwise, }\end{cases} \\
p \in P, i \in V, k=0, \ldots, K
\end{gathered}
$$

Eq. (1) is an objective function, which minimizes the summation of $\alpha_{k}$ for all stages. When $\alpha_{k}$ exceeds 1.0, we assume that traffic policing is applied at the edge node by multiplying $d_{p}$ by $1 / \alpha_{k}$. Eq. (2) is due to the flow conservation constraint for each stage $k$. Eq. (3) is a capacity constraint. If link $(i, j)$ has been repaired by the current stage $k$, Eq. (3) functions as the general capacity constraint; the summation of traffic included in link $(i, j)$ should not exceed $\alpha_{k} C_{i j}$. Otherwise, the right side becomes zero. Eqs. (4), (5), and (6) are physical links repairing constraints. Eqs. (4) and (5) represent that all links are bidirectional, and when a link is repaired, both directions are simultaneously repaired. Eq. (6) means that 


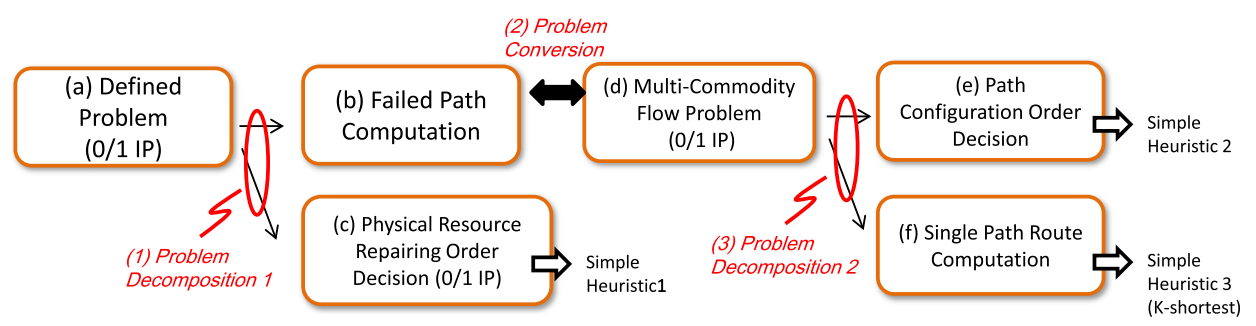

Fig. 1. Decomposition of complicated 0-1 IP problem

each link can be recovered once through all stages. Eqs. (7) and (8) are variables.

As represented by Eqs. (7) and (8), this problem is formulated as 0-1 ILP. Generally, 0-1 ILP is classified as NP-hard since the satisfiability problem (SAT) can be reduced to 0-1 ILP within polynomial time [5].

\section{Problem-decomposition method}

\subsection{Overview of problem-decomposition}

This section is on the problem-decomposition method for solving the problem by using simple heuristic algorithms within polynomial time. Fig. 1 illustrates our method.

The method first divides the (a) 0-1 ILP problem into two problems: (b) the failed path computation problem and (c) the physical resource repairing order decision problem. This division is based on our hypothesis: if each divided problem has the same objective function, the performance degradation caused by the problem decomposition is small.

For the (b) failed path computation problem is to determine $x_{i j k}^{p}, p \in P_{k}^{f}$. The set $P_{k}^{f}$ has the source and destination node pairs, which do not have a path between them at the beginning of stage $k$. By giving $d_{p}, p \in P_{k}^{f}$, and the available resources for those paths, the (b) failed path computation problem is defined as a (d) nonbifurcation multi-commodity problem. Though problem (d) is NP-hard [4], the path configuration order for recovery often accords with the network operation policy, e.g., the quality of services and/or service level agreement. Therefore, the (d) nonbifurcation multi-commodity problem is moreover divided into the (e) path configuration order decision problem and (f) single path route computation problem.

\subsection{Simple heuristics}

Each of the decomposed problems, (c), (e), and (f) can be solved by simple heuristic algorithms as follows.

1. Heuristic 1 for (c): for the set of failed links $(i, j) \in E^{f}$, the link is repaired in the descending order of $\sum_{p \in P} w^{p} d^{p} x_{i j k}^{p}$. The value $w^{p}$ is the priority of traffic
demand.

2. Heuristic 2 for (e): for the set of failed paths $p \in P_{k}^{f}$, the path is repaired in the descending order of $\mathrm{w}^{\mathrm{p}} d^{p}$.

3. Heuristic 3 for (f): the K-shortest path algorithm [6] is performed between $p \in P_{k}^{f}$. For each candidate path, it computes the maximum link utilization by assigning $d_{p}$. Then, it selects the best path, whose maximum link utilization is minimum. 


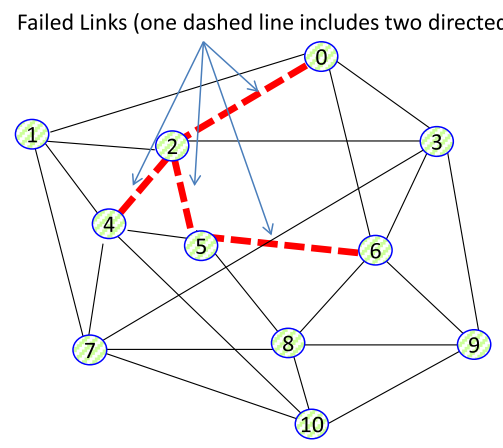

(a) Network Topology

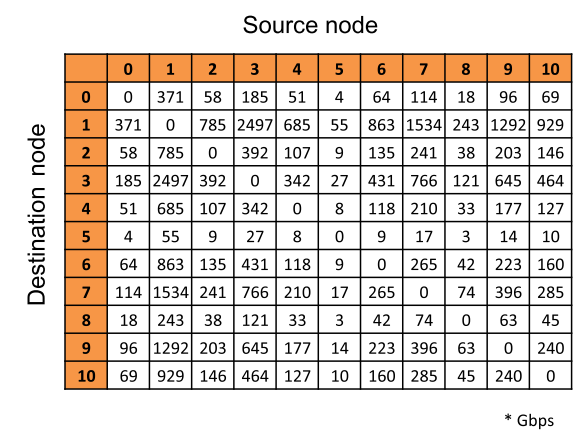

(b) Traffic Matrix

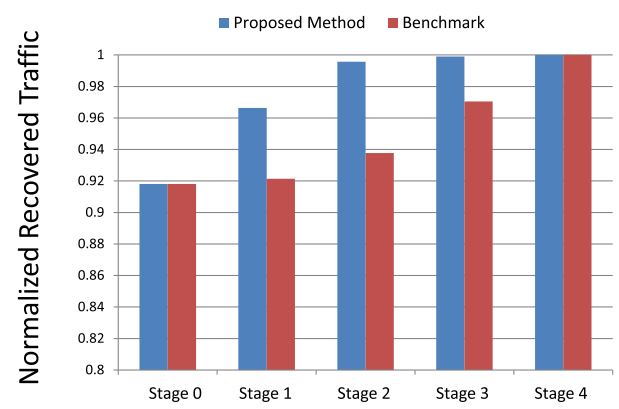

(c) Numerical Example

Fig. 2. Simulation topology, traffic and numerical example

For heuristics 1 and 2, it is obvious that repairing in the descending order of traffic amount is effective. To consider the characteristic of traffic, e.g., best effort, emergency call, we set the parameter $w^{p}$. For heuristic 3, we assume that there are a few numbers of routes between $p \in P_{k}^{f}$. Then, the best path from the K-shortest path algorithm becomes nearly optimal solution in most cases.

The computation order becomes polynomial. The bottleneck of computation is the heuristic 3. By using the typical Yen's algorithm, the computation becomes $O(k V(E+\log V))$, where $k$ is the number of computation paths [6].

\subsection{Numerical examples}

Fig. 2 illustrates the evaluation network topology and numerical examples. Note that the evaluation objective is proving our hypothesis that the problem decomposition is valid strategy if each divided problem has the same objective function, and showing the effectiveness on the basis of the simple heuristics. We do not make a strict comparison with the optimal upper bound by directly solving the problem in section 2 .

As shown in Fig. 2(a) and (b), the network has 11 nodes and 25 links, and traffic demands are given by the gravity model in accordance with the population. As first setup, the traffic is mapped to the network topology based on the shortest path routes. The link capacity $C_{i j}$ of the given network topology is set so that the utilization of all links becomes about $90 \%$ for clarifying the effect of repairing. The directed 8 links are failed at stage 0 , and both directions (two links) are simultaneously repaired at one stage, e.g., two links have been repaired at stage 1, and four links have been repaired at stage 2 . We use two methods for repairing of failed 
links and path routes: one is our simple heuristic method described in Sec. 3.2, and the other is the benchmark method, which repairs physical links randomly and computes the path routes by using the shortest paths algorithm. The evaluation index is the cumulative traffic amount, which is regulated as 1 when all links are repaired.

As shown in Fig. 2(c), our method gained 13\% of the whole traffic amount against the benchmark method: we believe that our hypothesis is correct in terms of practical use. Though we omit the results with other topology and traffic conditions because of the page limitation, we found that the performance mainly depends on the available resources; optimization is more effective when the network resources are well utilized before the failure or the scale of failure is large.

For the optimality of our method, we confirmed the validity of our heuristics 1 and 2 in a brute force test. For heuristic 3, it does not assure that the solution will be the upper bound before the problem decomposition. However, in this simulation, we found that the best path from the K-shortest algorithm and the shortest path become the same in many cases. This fact suggests that any path computation may produce almost the same solution in the non-bifurcation environment.

We do not discuss the quantitative computation time in this paper. The dominant computation is the $\mathrm{K}$-shortest path algorithm, and its computation time is about several tens of seconds in a practical environment [6].

\section{Conclusion}

For the traffic non-bifurcation environment, we defined the progressive disaster recovery problem as 0-1 ILP. Because the problem is classified as class NP-hard, we provide a problem decomposition method, where decomposed problems can be solved by simple heuristic algorithms, which achieves and improvement of $13 \%$ over the benchmark method. The objective of this paper is to be a flash report of the novel problem formulation and the way of problem decomposition. A strict comparison with the optimal upper bound that is made by directly solving the original problem is future work. 